\title{
Transcatheter arterial embolization for delayed recurrent intrathoracic bleeding caused by anastomotic leakage after minimally invasive McKeown esophagectomy
}

\section{Dong-lin LI}

Suining Central Hospital

Jie-wei HU

Suining Central Hospital

Hai-ning ZHOU ( $\nabla$ zhouhaining1976@163.com )

Department of thoracic Surgery, Suining Central Hospital, Suining city

\section{Case report}

Keywords: Transcatheter arterial embolization, Delayed recurrent intrathoracic bleeding, Anastomotic leakage, McKeown esophagectomy

Posted Date: January 14th, 2021

DOl: https://doi.org/10.21203/rs.3.rs-143777/v1

License: (c) (1) This work is licensed under a Creative Commons Attribution 4.0 International License.

Read Full License 


\section{Abstract}

Background: Anastomotic leakage (AL) is a serious complication after minimally invasive esophagectomy (MIE). Its development into delayed recurrent intrathoracic bleeding is very rare and may lead to fatal consequences.

Case presentation: We report a case of 67-year-old female who developed intrapleural infection, mediastinal infection and delayed recurrent intrathoracic bleeding as a complication of AL following minimally invasive McKeown esophagectomy. The triple antibiotics of imipenem and cilastatin sodium, vancomycin, and fluconazole were used for anti-infection treatment. Transcatheter embolization of subclavian artery branch vessel avoided re-bleeding. As a result, the patient recovered well and was discharged 58 days after initial surgery.

Conclusion: Delayed recurrent intrathoracic bleeding owing to $A L$ is a rare and life-threatening complication after MIE. Transcatheter arterial embolization (TAE) can provide a minimally invasive and effective method to control delayed recurrent intrathoracic bleeding after esophagectomy.

\section{Background}

The incidence of esophageal cancer is increasing recent decades, and the main treatment is surgery combined with neoadjuvant chemotherapy or neoadjuvant chemoradiation [1]. AL is a common complication following a minimally invasive McKeown esophagectomy, the severity of which ranges from asymptomatic to sepsis, accompanied by multiple organ failure, and is associated with high costs, a prolonged hospital stay, a serious deterioration in quality of life, and an increased mortality [1-2]. AL leading to delayed recurrent intrathoracic bleeding after MIE is a rare and life-threatening complication.

\section{Case Presentation}

A 67-year-old female patient with squamous cell carcinoma of the upper esophagus confirmed by endoscopy visited our Tertiary Medical Center. A minimally invasive McKeown esophagectomy with gastric conduit reconstruction and a left cervical anastomosis (circular stapler with a size of $23.5 \mathrm{~mm}$ ) was performed after multidisciplinary team meetings. The pathological tumour stage was pT2NOMO (AJCC, 8th edition). On postoperative day (POD) 7, the patient complained of chest pain and tightness, and purulent fluid was observed in the mediastinal drainage tube. The AL was confirmed by CT showing pneumomediastinum and mediastinitis, right-sided pleural effusion, and left cervical sinus formation (Fig. 1) and extravasation of contrast during a dynamic swallow study. We used cefoperazone and sulbactam sodium and levofloxacin to fight infection based on the susceptibility test of pus (Enterococcus faecalis) and open neck incisions for drainage. On POD 15, the triple antibiotics of imipenem and cilastatin sodium, vancomycin, and fluconazole were used because of the pus cultured Candida tropicalis, repeated fever and continued high levels of white blood cell counts. 
On POD 19 and 21, the patient experienced two intrathoracic bleeding. $540 \mathrm{ml}$ and $300 \mathrm{ml}$ of bloody fluid were drained from the mediastinal drainage tube, which lasted 90 minutes and 30 minutes, respectively. The patient had an immediate chest CT examination, which failed to locate the source of bleeding. What's worse, on POD 24, another intrathoracic bleeding occurred, about $700 \mathrm{ml}$ of bloody fluid was drainaged from the mediastinal drainage tube. An enhanced multi-slice chest CT scan was performed immediately, in the upper mediastinum area, a curved, slightly enlarged blood vessel with a diameter of about $3 \mathrm{~mm}$ caught our attention (Fig. 2). Because of a strong clinical suspicion of ongoing bleeding, the patient was emergently taken to the Hybrid operating rooms while having massive transfusion protocol after the patient's family signed an informed consent. Angiography was performed using an interventional angiography system (Artis zeego, Germany). Two experienced interventional radiologists performed endovascular procedure. The patient took the supine position and a 5-Fr introducer sheath (TERUMO, Tokyo, Japan) was inserted into the right common femoral artery under ultrasound guidance through the Seldinger procedure. Selective angiography of the right subclavian artery was performed and revealed an approximately $3 \mathrm{~mm}$, curved, irregular blood vessel originated from the right subclavian artery (Fig. 3). Based on CT and angiographic findings, we considered this curved blood vessel to be the culprit causing recurrent intrathoracic bleeding, although the extravasation of the contrast agent was not obvious. Superselective embolization of the right subclavian artery branch vessel with polyvinyl alcohol particles (100-300um) and gelatin sponge (350-560um) was performed. No active bleeding was seen on re-angiography. Since then, the patient had never experienced intrathoracic bleeding again. Antibiotic treatment was continued for a total of 14 days. Laboratory and radiographic evolution paralleled the favourable clinical recovery. The patient was discharged 58 days after initial surgery.

\section{Discussion}

Treatment for esophageal cancer usually consists of surgery combined with perioperative chemical (radio) therapy [1]. The incidence of AL after McKeown esophagectomy remains relatively high, which may be related to tube gastric perfusion, anastomotic tension, construction of the anastomosis [1, 2]. The Esophageal Complications Consensus (ECCG) classified AL into three types, typ I: local defect requiring no change in therapy or treated medically or with dietary modification; typ II: localized defect requiring interventional (not surgical) therapy, for example, drain placement or stenting; typ III: localized defect necessitates surgical therapy [3]. Measures for prevention of anastomotic leak include reducing the tension of the anastomosis, avoiding damage to the vascular arch, strengthening the anastomosis and wrapping anastomosis with omentum [2].

Intrathoracic hemorrhage is a common clinical emergency and requires a significant health and economic burden, the source of which varies [4]. Early postoperative intrathoracic bleeding after esophagectomy, especially with intrathoracic anastomosis, resulting from mechanical anastomotic failure or vascular injury [4]. In this case, the branch vessels of the subclavian artery may be damaged during esophagectomy. The physiological response of these small blood vessels to tearing is posttraumatic contraction and thrombosis secondary to hemostasis. However, owing to $\mathrm{AL}$, the combination 
of the long-term erosion of pus and the shedding of necrotic tissue are the presumed initiating factors of the pathological cascade of delayed recurrent intrathoracic hemorrhage.

In most patients, traditionally, an additional thoracotomy is considered the firstline therapeutic option to control the blood loss. However, in our case, a re-thoracotomy to control bleeding can be difficult and tedious, owing to severe infection, the anatomic inaccessibility caused by extensive adhesion and limited exposure of the thoracic roof. On the contrary, TAE is minimally invasive, facilitates the identification of bleeding sources (extravasation or irregular arterial walls) through angiography, and can effectively achieve hemostasis [5]. Moore $\mathrm{C}$ et al. had extended this technique to the thoracic cavity by using selective thoracic aortography with transcatheter embolization of intercostal artery, providing a minimally invasive auxiliary method for the treatment of refractory intrathoracic hemorrhage [6]. Apart from the direct sign of contrast extravasation on angiography, irregular blood vessels, cut off blood vessels, arteriovenous shunts, pseudoaneurysms, increased new blood vessels and dilated arterioles were also considered signs of bleeding indicating embolization [7]. In this case, a selective angiography can visualize a curved, irregular blood vessel originated from the right subclavian artery, and the microcatheter was used to embolize the bleeding artery as distal as possible. If the source of bleeding cannot be identified angiographically, empiric embolization or blind embolization can be considered. Empiric embolization technique has been shown no difference in clinical outcome when compared with embolization of an identifiable source of bleeding [8]. Microcoils, polyvinyl alcohol particles, NBCA, vascular plugs, and gelatin sponges are used as embolic agents. Interventional radiologists should fully consider the unique characteristics and embolization mechanism of each embolic agent, and select the appropriate embolic agent based on collateral network, vascular lesions, cost and operator skills [5].

\section{Conclusion}

Delayed recurrent intrathoracic bleeding caused by $\mathrm{AL}$ is a rare and life-threatening complication after MIE. Transcatheter arterial embolization techniques, while not a replacement for thoracotomy, may provide a minimally invasive adjunct for control of bleeding. More studies are required to confirm the applicability of this approach as a suitable option to address this difficult clinical challenge, particularly after intrathoracic surgery.

\section{Abbreviations}

AL

Anastomotic leakage

MIE

Minimally invasive esophagectomy

TAE

Transcatheter arterial embolization

\section{AJCC}

American Joint Committee on Cancer 
POD

Postoperative day

ECCG

Esophagectomy Complications Consensus Group

\section{Declarations}

\section{Acknowledgements}

The authors thank the interventional radiologists for their contribution to this report.

\section{Authors' contributions}

All authors contributed to the study concept or design, data collection, data analysis and interpretation, and writing of the paper.

\section{Funding}

Not applicable.

\section{Availability of data and materials}

The data used in this report are available from the corresponding author.

\section{Ethics approval and consent to participate}

Our institution Ethics Committee approved the study.

\section{Consent for publication}

Written informed consent was obtained from the patient and her son for publication of this case report and accompanying images.

\section{Competing interests}

No competing interests.

\section{References}

1. Jezerskyte E, Saadeh LM, Hagens ERC, Sprangers MAG, Noteboom L, van Laarhoven HWM, et al. Long-term health-related quality of life after McKeown and Ivor Lewis esophagectomy for esophageal carcinoma. Diseases of the Esophagus. 2020.

2. Verstegen MHP, Bouwense SAW, van Workum F, Ten Broek R, Siersema PD, Rovers M, et al. Management of intrathoracic and cervical anastomotic leakage after esophagectomy for esophageal cancer: a systematic review. World Journal of Emergency Surgery. 2019; 14(1). 
3. Low DE, Alderson D, Cecconello I, Chang AC, Darling GE, D'Journo XB, et al. International Consensus on Standardization of Data Collection for Complications Associated With Esophagectomy: Esophagectomy Complications Consensus Group (ECCG). Ann Surg 2015; 262:286-94.

4. Javed A, Pal S, Chaubal GN, Sahni P, Chattopadhyay TK. Management and Outcome of Intrathoracic Bleeding due to Vascular Injury During Transhiatal Esophagectomy. Journal of Gastrointestinal Surgery. 2011; 15:262-266.

5. Chatani S, Inoue A, Ohta S, et al. Transcatheter Arterial Embolization for Postoperative Bleeding Following Abdominal Surgery. CardioVascular and Interventional Radiology. 2018; 41:1346-1355

6. Moore C, Kwayisi G, Esiobu P, Bashan-Gilzenrat KA, Matthews LR, Nguyen J, et al. Successful treatment of massive hemothorax with class IV shock using aortography with transcatheter embolization of actively bleeding posterior left intercostal arteries after penetrating left chest trauma: A case for the hybrid OR. International Journal of Surgery Case Reports. 2018; 48:109-112.

7. Shin JH. Refractory gastrointestinal bleeding: role of angiographic intervention. Clin Endosc. 2013; 46(5):486-91.

8. Loffroy R, Guiu B, D'Athis P, Mezzetta L, Gagnaire A, Jouve JL, et al. Arterial embolotherapy for endoscopically unmanageable acute gastroduodenal hemorrhage: predictors of early rebleeding. Clin Gastroenterol Hepatol. 2009; 7:515-23.

\section{Figures}



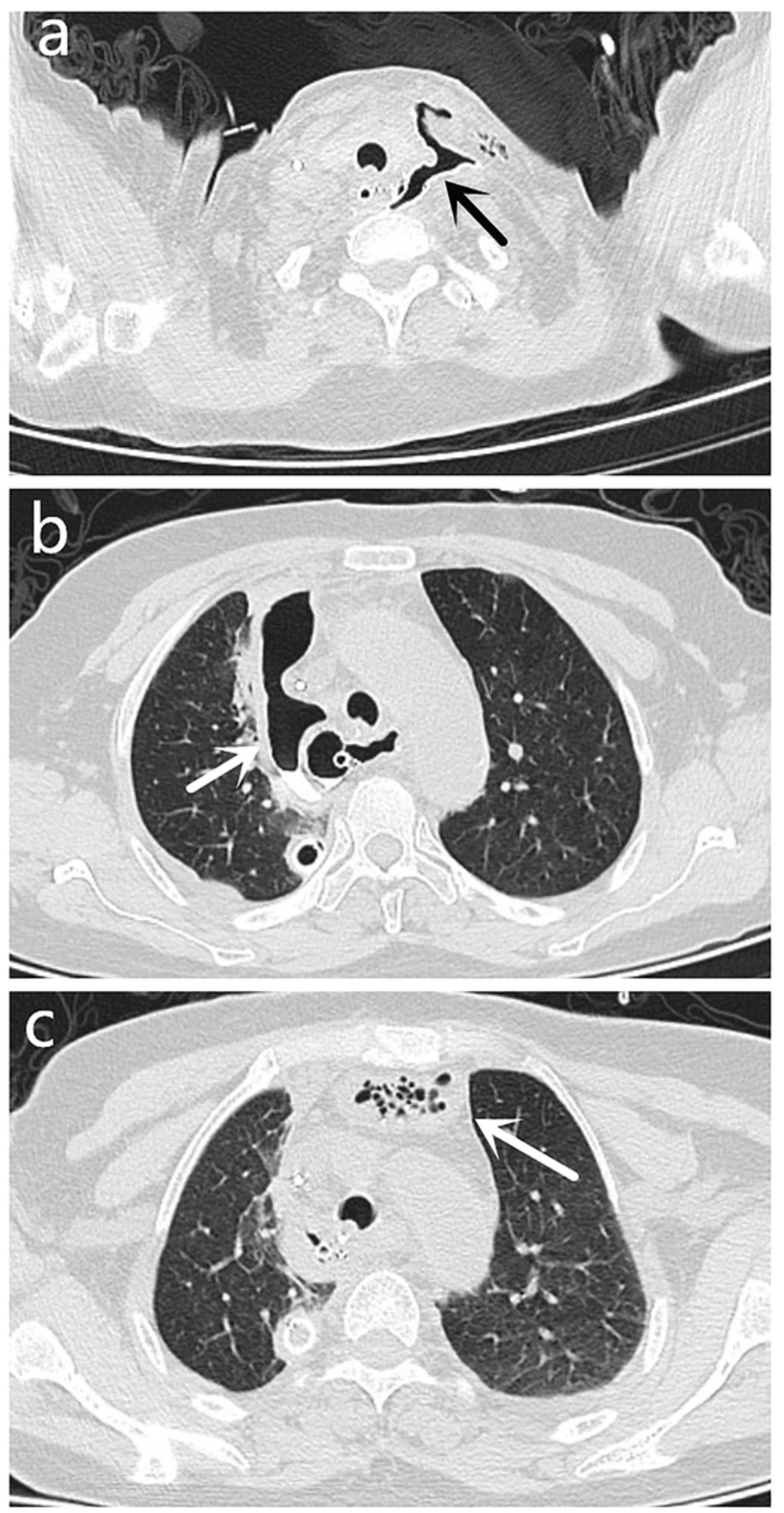

Figure 1

CT manifestation of anastomotic leakage. 

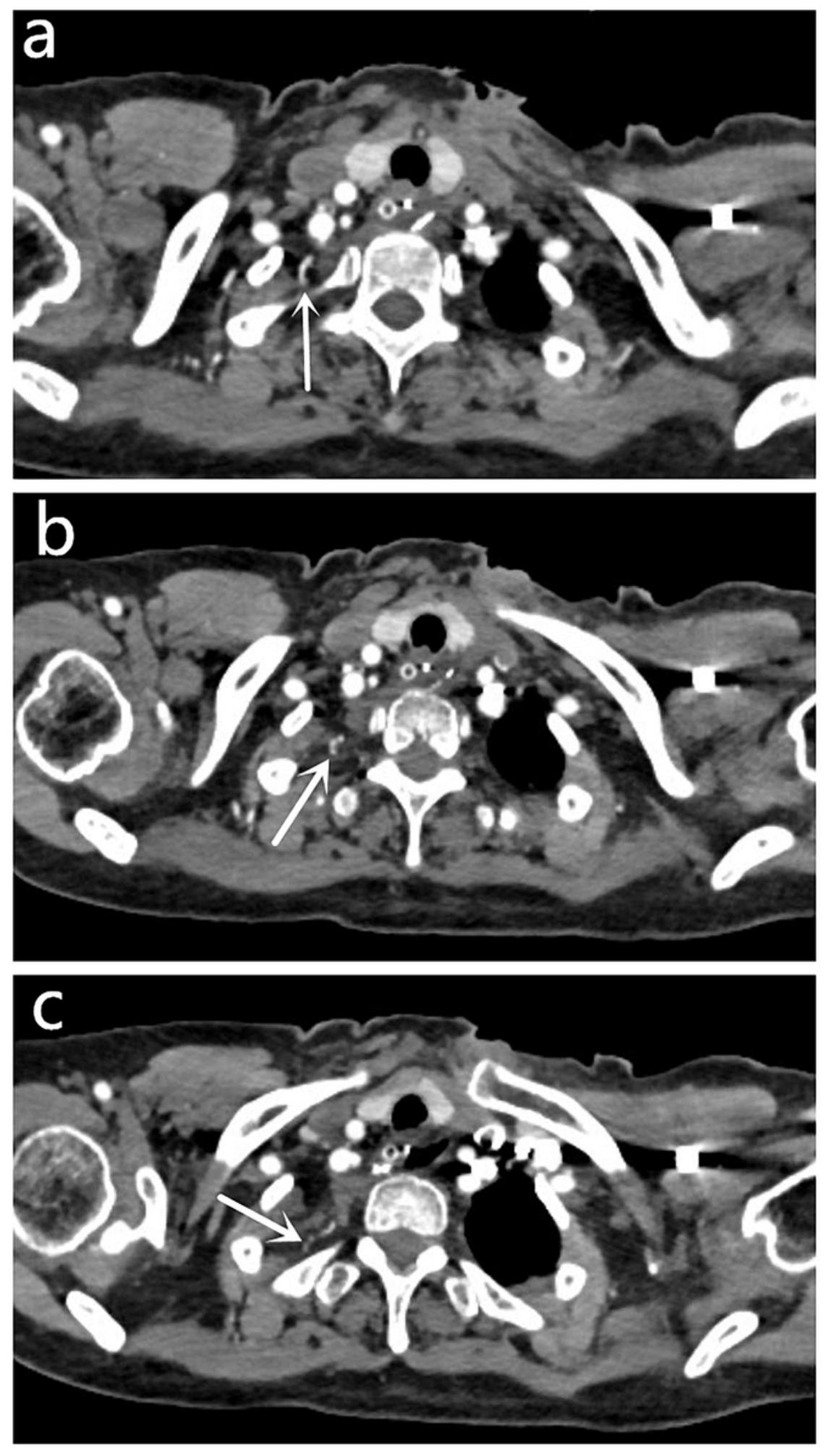

Figure 2

Enhanced multi-slice chest CT manifestation of a bleeding artery originating from the branch of the right subclavian artery. 

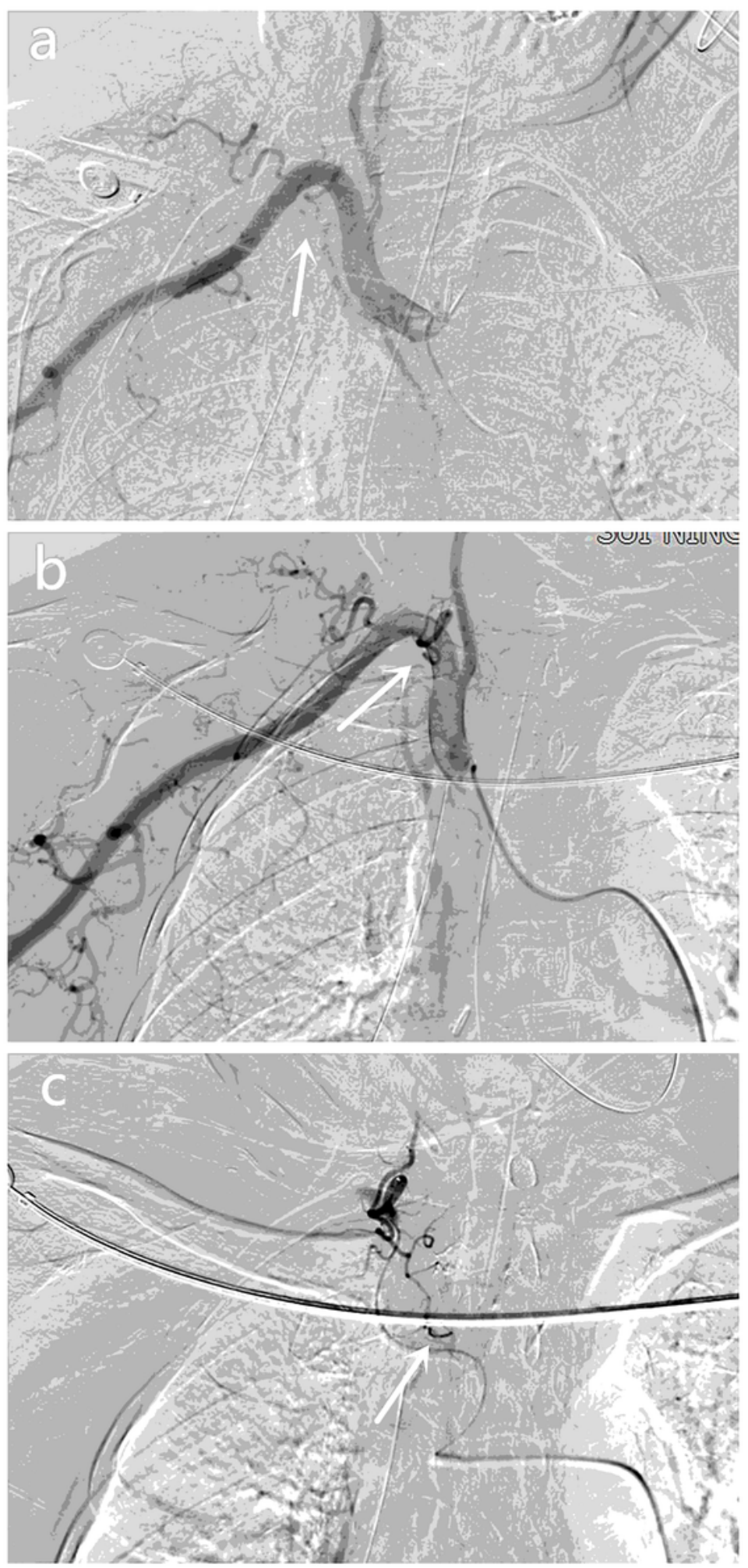

Figure 3

Findings of selective right subclavian angiography.

\section{Supplementary Files}

This is a list of supplementary files associated with this preprint. Click to download. 
- CAREchecklistEnglish2013.pdf

Page 10/10 